\title{
Entre certidumbre e incertidumbre:
}

Semi narios*

Between certainty and uncertainty:

Academi c research

* Investigaciones académicas realizadas por estudiantes de Quinto Año de Arquitectura de la Universidad de Chile, bajo la dirección de un profesor guía.

\section{Dга. Магі́ B Ber†гаnd S.}

\section{"Resumen"}

Primer viaje por los caminos y paisajes de la vida del "saber", seminario es, simultáneamente, viaje y relato justo comunicable a terceros. Viajes y relatos, viajeros y logros consecuentes con el mundo cognitivo implicado por la inflexión inicial determinante de maneras de viajar y maneras de relatar. Viajes inscritos en el avance general del saber hacia "complejidad" que enlaza certidumbre a indeterminación a incertidumbre a indecible y a aporías en mundos de saber-poder o en mundos de saber-hospitalidad. Mayoritariamente, los seminarios buscan acceder al "saber-hospitalidad" que indaga la "concretitud" de lo que ya "está ahí". en acto, cotidiano: manifiestan, en otros términos, la urgencia de cultivar medios, recursos, condiciones para recorrer caminos atentos al "rostro" Levinasiano de la realidad.

\section{"Abstract"}

The first voyage upon the paths and I andscapes in the Iife of "knowl edge"; students' research papers are si multaneously a voyage and just a tale told to others. Voyages and tales, travelers and achi evements that correspond to the cognitive world implied by the inflection determined by the means of traveling and the means of telling. These voyages are inscribed in the path towards "compl exi ty" that links certainty to indeterm nation to uncertainty to unspeakable to "aporias" in worlds of knowl edge-power or worlds of knowl edge-hospitability. Most of the commented students' research papers seek the "knowl edge-hospitability" that i nvestigates the concretion of what is actually al ready "there", the quotidian: in other words, they manifest the urgency to cultivate means, resources and ways to travel across paths of knowl edge while consi dering the "Levi nasian" façade of real ity.

"Palabras clave"

EPISTEMOLOGÍA, CERTIDUMBRE/INCERTIDUMBRE POÉTICA, COMPLEJIDAD, EXPERIENCIA, REPRESENTACIÓN

"Key words"

EPI STEMOLOGY, CERTAI NTY/ UNCERTAI NTY, POETI CS, COMPLEXI TY, EXPERI ENCE, REPRESENTATI ON

\section{Ргіmer viaje...}

Primer viaje por los caminos y paisajes de la vida del "saber"1 el seminario es, simultáneamente, viajar, partir, embarcarse y es, también, decantar el viaje como relato justo comunicable a terceros. Viajes irremediablemente consecuentes con los mundos cognitivos donde se encuentran los caminos, paisajes y eventos de lo indagado: consecuentes con el mundo de cada "manera de saber", por ejmeplo con lo que, en cada caso, se determina como "saber" - logos².

BERTRAND SUAZO, María. "Entre certidumbre e incertidumbre: Seminarios". Revista De Arquitectura. Noviembre de 2005. Pág. 12.

Dudamos de las implicaciones de la noción de capital social para la teoría y la práctica del desarrollo porque neutraliza el análisis de la inequidad. Focalizada sólo en comunidades pobres reducidas a variables cuantificables universales, la noción instala un punto ciego que excluye o descalifica el rol de los sistemas subyacentes.

Reconocer puntos ciegos evita reiterarlos al investigar y al derivar políticas de desarrollo. K.A. Schafft, D.L. Brown, 2003. Mi traducción.

M. Bertrand, 2004; I. Stengers, 2002; J. Derrida, 1996; D. Schindler, 2003; C.Z. Elgin, 2002.

2 D. Dickson, 2001; E. Lévinas, 1971; I. Prigogine,I.Stengers, 1979-1986; I. Stengers, op. cit.; D. Schindler, op. cit. 
Viajes, viajeros y, en suma, logros consecuentes con el mundo cognitivo recorrido. Así, hay viajes y relatos de viajes que invocando sólo términos, sólo vocablos, de la "ciencia de lo complejo", caen en puntos ciegos ${ }^{3}$ porque transcurren íntegramente en alguno de los mundos cognitivos del "espejismo universal" de la ciencia clásica donde, necesariamente, los viajeros recorren caminos unidireccionales, registran organizaciones determinista de objetos y trayectorias predecibles en espacios lisos ${ }^{4}$ euclidianos cuyo "tiempo" equivale a medición de cambios de posición o de estado ${ }^{5}$. Indagados en mundos y dinámicas cognitivas ajenas a devenir, a imprevisible, a diversidad cualitativa de la "ciencia de lo complejo", los términos invocados no viajan ni hacen partir: su propia inconsecuencia al momento de instalar sus "espacios de representación"6 cierra el acceso a la vida, al pulso-tónico ${ }^{7}$ a los descubrimientos y riesgos de la poética del saber ${ }^{8}$.

\section{Inflexión inicial}

El inicio del viaje: es crucial el momento que instala los "Espacios de Representación" Andlerianos consecuentes, coherentes ${ }^{9}$ en epistemologías "más fuertes" 10 que las de los escenarios especulares simétricos examinados por M. Foucault. La naturaleza epistémica y teórica de lo que se instala va a implicar: las maneras de partir, viajar y relatar, los recorridos, las visitas y las estadías cognitivas... así como los manejos de la tensión, tónica, "realidad"/representación"11.
Pero hay más. Los avances generales y sectoriales de la vida del saber hacia la "ciencia de la complejidad"12 reconfiguran el entendimiento de logos desde el momento que lo prolongan a sus efectos ${ }^{13}$, lo articulan a cultura, filosofía, artes ${ }^{14}$, lo reconocen inmerso en la "flecha del tiempo" 15 y emanando de puntos de enunciación otros determinados por nuevos "tejidos" sujeto/objeto,

individual/societal, adentro/afuera, objetivo/ subjetivo ${ }^{16}$. Habitan mundos de certidumbre, indeterminación, incertidumbres, indecibles, aporías.

Los avances de la vida del saber piden, en suma, ir "mas allá": no contentarse con satisfacer las solas condiciones de rigor lógico, pragmático, lingüístico y epistémico internas, intrínsecas a cada viaje, sino reconocer, además, la condición de "operación" situada, relativa del Espacio de Representación ${ }^{17}$, la necesidad de crear los "idiomas" del viaje ${ }^{18}$ y de aceptar que el encuentro, inevitable, con cuotas de aporías, indeterminaciones, incertidumbres, indecibles... forma parte del saber indagado. Ir "más allá" indispensable para que el saber re-encuentre -responsablemente- sus consecuencias, sus efectos en la complejidad y devenir de la realidad ${ }^{19}$ : ir "mas allá" que deviene condición sine qua non del saber de Arquitectura y de Urbanismo.

\section{Dialógicas y re-encuentros}

Esos avances introducen renovaciones y re-configuraciones epistémicas, conceptuales, metodológicas intensas: la "ciencia de lo complejo" instala flujos y fuerzas, bifurcaciones, orden por fluctuaciones, incertidumbre... que inician el reencuentro con el aquí-ahora conjuntamente con sus temporalidades, actores, eficacias, narrativas e indeterminaciones: Io "re-encanta" 20. Los intangibles, lo cualitativo, lo subjetivo... los expulsados, en suma, de la "ciencia clásica" retornan renovando y revisitando las representaciones canónicas y los "discursos" del saber: ciudad 21, "espacio", "tiempo", "persona", diversidad, complejidad, devenir, equidad...; estructuralismo, modelizaciones racionalistas, empiristas, fenomenológicas... nada queda indemne ${ }^{22} \ldots$

Así, el saber deviene manejo atento, abierto, preciso, responsable de las dialógicas "realidad" / representación: deviene instalación de esa tensión en mundos epistémicos consecuentes, coherentes con lo indagado. Y qué ocurre: el reencuentro del saber prolongado a los efectos de sus acciones en la acción y vida cotidiana ${ }^{23}$ revela mundos cognitivos -epistemologías- con distintas maneras de relacionarse con "lo real".

\section{Saber-máquina de guerгa / Saber-hospitalidad}

Porque hay mundos y mundos del saber. Hay mundos cognitivos -demasiado numerosos según $B$. Latour-que inscriben desde el inicio al viajero y al viaje en la "bifurcación separadora"24 del "saber" como violencia de poder: mundos del saber como "fuerza" Weiliana que embarcan en "máquinas de guerra": en enunciados que imponen, no

\footnotetext{
3 I. Prigogine; I. Stengers; op. cit.

G. Deleuze; F. Guattari, 1980.

I. Prigogine; I. Stengers; op. cit.

D. Andler, 1987.

J-L. Nancy, 2000-2003.

P. Feyerabend, 1975-1979; E. Lévinas, 1991; M. Onfray, 1993.

9 I. Stengers,

M. Serres, 1980.

M. Bertrand, 2005a, 2005b; M. Serres, 1980, 1992; I. Stengers; op. cit.; J. Derrida; op. cit.; M. Onfray; op. cit.; M. de Certeau, $1975 ;$ E. Lévinas, 1991.

2 F. Dosse, 1995, 1997; E. Montuschi, 2004; J. Popke 2003; J-M. Paige, 1999; F. Farinelli, 1998.

M. Serres, 2001, 2003

14 C.Z. Elgin; op. cit.

15 I. Progogine; op. cit.; I. Stengers, 2003

16 I. Aravot, 2002; R. Kallus, 2001; A. Latham, 2003; J. Okely, 2001

17 M. de Certeau; op. cit.

18 I. Stengers, 2003

19 B. Latour; op. cit.; J.C. Clingermayer, 2004; M. Serres, 2003.

La fuerza es lo que hace una cosa de cualquiera que le esté sometido. [..] El poder que posee de transformar a los hombres en cosa es doble y se ejerce en dos sentidos; petrifica de forma diferente, pero tanto a unos como a otros, las almas de quiene la sufren y de quienes la manejan... S. Weil, 1953-2005.

20 I. Stengers, 2003.

21 A Latham; D.P. McCormack, 2004; H. Lefevbre, 1986; R. Kallus; op cit. I. Aravot; op cit.

22 M. Bertrand, 2005a y 2005b.

23 D. Schindler; op. cit.; E. Lévinas.

24 I. Stengers, 2003
} 
escuchan, rompen, expulsan ${ }^{25}$. Saberes que declaran una cosa mientras operan otra ${ }^{26}$, que transforman todo y a todos en "cosa" manipulable ${ }^{27}$ : mundos -por ejemplo, suelos epistémicos- fundacionalmente consagrados al saber como poder.

Pero también están los mundos cognitivos de la "hospitalidad del saber" y del saber como hospitalidad. Hasta ahora, los mundos epistémicos de más alta complejidad y los más ricos en exigencia y en controladores internos y externos. Mundos politéticos. Tal vez, por lo mismo, menos transitados. Porque no son mundos para viajes de algunas semanas sino para aventuras de más largo aliento. En ellos, la cognición-acogida, el saber-hospitalidad que escucha e implica la pluralidad de dimensiones del entorno de personas exige Espacios de representación idénticamente complejos, dinámicos: por ejemplo, exige idiomas, lenguajes, operatorias, otros $^{28}$, consecuentes y coherentes con lo indagado. Los viajes por los mundos del saberhospitalidad, dialógicos, pacificadores parecen ser los más adecuados para que el saber de Arquitectura, de Urbanismo re-encante la "concretitud de lo real" 29

Mundos del saber-poder, mundos de saber-hospitalidad: cada una de las opciones del viaje del saber pone a prueba el ser del viajero y manifiesta los diferentes límites y distintos contenidos de la "verdad" que permiten indagar. Uno y otro revelan, no obstante, que el verdadero viajero, que el verdadero tema de cada relato de viaje es, en último término, la poética que va a constituir el saber como poder o como hospitalidad. La tensión, a veces intensa, viajes-relato de algunos seminarios sintomatiza la presencia del verdadero viajero, del verdadero tema así como la primera puesta a prueba ante el tipo de saber indagado ${ }^{30}$.

\section{Hacia la complejidad}

Las obligaciones internas y contextuales ${ }^{31}$ que enmarcan los objetivos y decisiones Arquitectónicas o Urbanísticas inscriben su saber en las epistémologías del saberhospitalidad: por ejemplo, en la avanzada de la complejidad. Le exigen, en otros términos, disponer de los medios, recursos y condiciones para realizar viajes extremos y para inscribir relatos singulares. Remitirse a enunciar logos como pensar íntegro ${ }^{32}$ abarcando el evento fenomenal percepción/cognición y su prolongación en "actuar justo" emoción/acción que externaliza -re-integrando al mundo- el producto de su actividad mental ${ }^{33}$ no basta: ese logos existe cuando, disponiendo de medios y recursos, viaja y escribe su relato. Sobre todo porque el viaje de este logos es aventura, es movimiento cognitivo habitado por la creación-construcción de su propia manifestación, poética que no es, nunca, ni segunda ni accidental sino constitutiva del pulso viajar-manifestar y del relato testimonial comunicable a terceros. Constitutiva del rigor del viajar y de la escritura que embarca a nuevos viajes ${ }^{34}$

El primer paso "seminario" -medido, cauteloso, controlado- reconoce y ensaya el pulso, caminos, viajes de cada viajero. Ensaya la respiración, hermeneútica, "concretitud de lo real" / representación ${ }^{35}$ recorriendo rutas a veces insospechadas, a veces demasiado pródigas en incertidumbre, indecibles, aporías. Desemboca, re-descubre o encuentra por primera vez situaciones, eventos de lo real que no lograba "conocer" ni manifestar antes de recorrerlos, visitarlos, contemplarlos o interpelarlos gracias a ese viaje ${ }^{36}$.

\section{Viajar-геlatar}

El primer paso constata que viajar es más que recorrer: es visitar ${ }^{37}$ y es habitar los eventos cognitivos del viaje y la escritura de los relatos que los hacen existir como saber preciso, riguroso y, sobretodo, coherente ${ }^{38}$. El primer paso verifica una y otra vez que ese avanzar habitando la manifestación de logos pide contar con medios, recursos y operaciones específicas a cada instancia realidad/representación y a cada momento de escritura como comunicación del saber constata las limitaciones, inconsecuencias, rigideces de medios que instalan puntos ciegos así como los vacíos, las carencias que no le permiten viajar como y a dónde desea ${ }^{39}$.

Y no sólo eso: el primer paso del seminario comprueba también que la poïesis de esas diferentes tensiones es afectada por la "flecha del tiempo": que viajar no se reduce a acumulación unidireccional, secuencial, plana, lisa sino que pasa a ser implicación ${ }^{40}$, proceso, devenir, enriquecimiento. Manera laberíntica de proseguir y de vivir ${ }^{41}$ donde los puntos de inflexión y las resistencias al sentido y a la representación fuerzan a elegir entre distintos caminos, cada uno de los cuales obliga a crear el idioma y los lenguajes de esa poïesis ${ }^{42}$ sin traicionarla, sin deformarla. Sin falsearla. Constatación que, inicialmente, desconcierta

25 B. Latour, 2004; E. Lévinas; op. cit.

26 I. Stengers, 2003.

27 I. Stengers; op cit: M. Serres; op cit. M. Bertrand, 2005a.

¿Estamos aquí tal vez para decir: casa, puente, fuente, puerta, balde, árbol frutal, ventana - o, incluso, pilar, campanario... pero, para decir, comprende, para decir así, como nunca antes las cosas creyeron SER tan intensamente? Novena Elegía de Duino. R.M. Rilke. Mi traducción.

28 H. Couclelis, 2003; M. Boumans; A. Beaulieu, 2004

29 B. Latour; op. cit.; E. Lévinas; op. cit.; G. Deleuze; F. Guattari; op. cit.; I. Stengers; op. cit.; B. Massumi, 2000.

M.J. Cazorso, 2003; C. Mellado, 2002; L. Figueroa, 2002; P. Tonacca, 2005; J.F. Vargas, 2004.

1 M. Bertrand, 2005a, S. Weil, 1949, E. Lévinas; op. cit.

32 O logos en tanto phronesis Heraclitiana, revelación Levinasia, coherencia de la comprensión Stengeriana...

33 D. Schindler; op. cit.; Stengers; op. cit.

Revelacion-escritura que avanza a grandes pasos al mismo tiempo que... "da vueltas como alguien extraviado en un bosque, volviendo sobre sus pasos, partiendo otra vez engañado (¿० guiado?) por el parecido de ciertos lugares, sin embargo diferentes, que cree reconocer...". (C. Simon, 1970).

34 I. Stengers, 2002; P. Feyerabend, 1997; D. Schindler; op. cit.

35 E. Lévinas, 1991.

36 J. Derrida, 1996; E. Bronfen, 2000; D. Sepúlveda, 2005; A.N. Urbina, 2005; J.F. Vergara, 2004

37 M Serres.

38 P. Toledo, 2002; R. Encina, 2003; F-J. Moure, 2003; A.M. de León, 2003; M. P. Silva; F. Rojas, 2004

39 M.J. Cazorso, 2003; F.I. Romero, 2003

40 K. Andersen; P. Solís, 2004; D. Sepúlveda, 2005

41 I. Stengers; op. cit.; D. Andler, 1987; M. Serres, 1985; M. Bertrand, 2004; A. Barrico, 1993-1998; K. Jenkins, 2003.

El estilo... está en relación con el estilete, ese pequeño cincel contemporáneo de las escrituras en superficies blandas. Una herramienta, prolongación del alma, instrumento del espíritu, mediación entre interior y exterior... M. Onfray. Mi traducción.

42 F.I. Romero; op. cit.; M.J. Cazorso; op. cit.; R. Pinto, 2005. 
y luego abre a la creación consecuente, coherente, de las representaciones de lo indagado.

Cuando eso ocurre, la "escucha" atenta del relato deja filtrar el trasfondo de rumores y de voces, de tumultos y de silencios determinantes del sentido de lo indagado. $Y$ el caminar de la escritura revive el ritmo del caminar seguro o incierto de cada etapa del viaje: lo escrito manifiesta el pulso de la vida del saber durante ese viaje. Pulso que anima, literalmente, un pequeño puñado de textos, excepcionales, del total de cincuenta y cuatro seminarios acompañados como segunda persona ${ }^{43}$, escuchando el pulso de los mundos encontrados-creados por el viaje, dejando ser $^{44}$ al indicar maneras de viajar. Sabiendo que, a la manera del "estilo" Onfrayano o del investigar Feyerabendiano, el viaje de construccióncreación cognitiva deviene, simultáneamente, "construcción de sí"45.

¿Qué hizo viajar?: mayoritariamente, búsqueda de mayor potencialidad dialógica y mayor inmersión en lo cotidiano del saber de Arquitectura, de Urbanismo. Particularmente en las prolongaciones de sus efectos sobre la realidad. Salvo excepción, el interés que se concentra, intuitivamente, en la búsqueda del suelo epistémico, los Espacios de Representación y los controladores de un saber riguroso, exigente y responsable perteneciente al mundo de logos-hospitalidad enfrenta, permanentemente, a la limitación de los medios teóricos, conceptuales, metodológicos y epistemológicos necesarios para materializar el viaje y la indagación deseados. Por lo mismo los viajes son breves, medidos, cautelosos aunque no carecen de todo riesgo cognitivo ${ }^{46}$ ni eluden la complejidad $^{47}$. Pero la amplitud del pulso, la

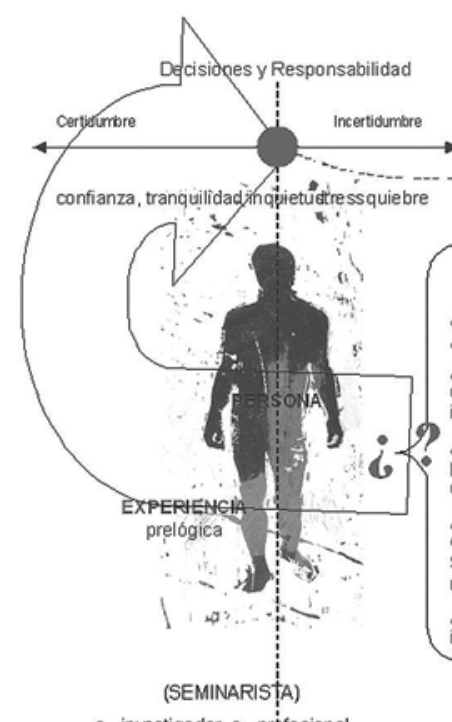

0 ...investigador, 0 ...proffesiona Persona singular. cegueras própias. Momento y lugar de vida: contexto de discursos,
valores, motivaciones. Hacia el Titulo, familia, trabajo, propia búsqueda:
Dinámicas certidumbre-incertidumbre.

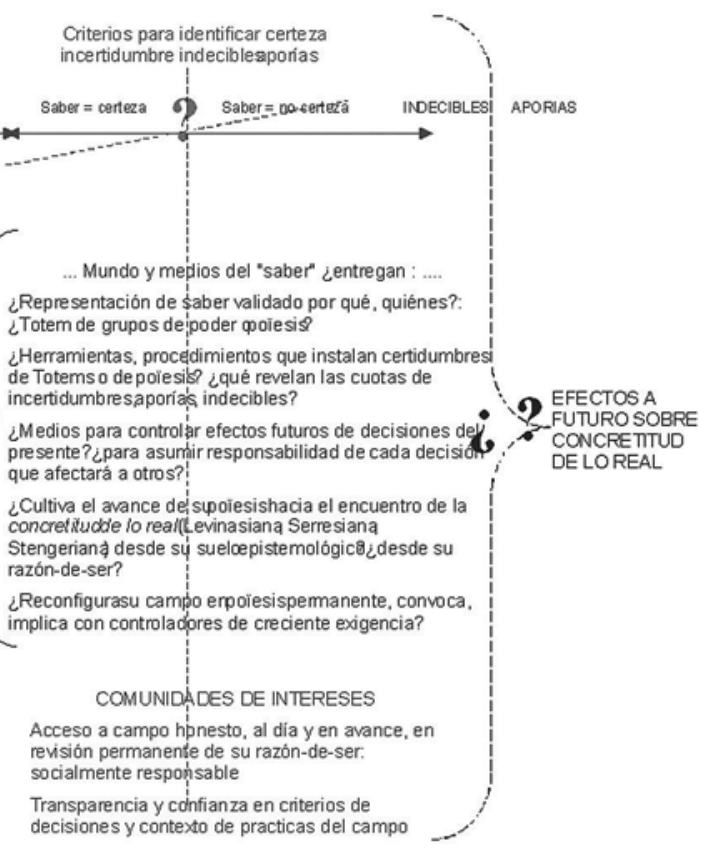

DINAMICAS CERTIDUMBFE - INCERTIDUMBRE

43 F. Varela, 1999; I. Stengers; op. cit.

44 E. Lévinas; op. cit.; J. Derrida, 1997; I. Stengers; op. cit.

45 M. Onfray; op. cit.; P. Feyerabend; op. cit.

46 R. Encina; op. cit.

47 M.J. Cazorso; op. cit.; I. García, 2002; A. de León; op. cit. 
profundidad y riqueza del saber, buscados, intuitivamente, a partir de la "concretitud de lo real" necesitó disponer y manejar con soltura medios más fuertes enmarcados por controladores -intrínsecos y contextuales- que exigen idiomas -estadísticos, probabilísticos, grafos, representaciones fuzzy, tónicas de escritura, cartográficos, fílmicos- consecuentes con lo indagado.

Búsqueda coherente con la intensificación de las exigencias de confiabilidad de las decisiones, que aumenta a medida que se avanza hacia sociedades fragmentadas ${ }^{48}$, individualistas, multiculturales ${ }^{49}$.

Particularmente en el caso de decisiones con fuerte impacto político relativas al "bien público" local ${ }^{50}$ donde la consideración de complejidad, devenir, evento, punto de bifurcación, fluctuación, crisis, riesgo... pide medios que permitan instalar representaciones de certeza-incertidumbre diferentes de las predicciones de riesgos inmobiliarios de megaproyectos urbanos ${ }^{51}$ : pide, más radicalmente, reconfiguración del "saber" desde el existir cotidiano ${ }^{52}$

\section{Certeza: saber-hospitalidad}

Mayoritariamente, los seminarios buscan acceder al "saber-hospitalidad": al saber que exige indagar la "concretitud" de lo que ya "está ahí", en acto, familiar, cotidiano -por lo mismo, "in-visible", enigmático-... desde, en esa concretitud ${ }^{53}$ en vez de, bifurcante, indagar -externamente- sobre ella. Inmersión que enfrenta a la exigencia de "representarlos" en su cotidianeidad ${ }^{54}$ en las aventuras de la "ruina de la representación" Lévinasiana ${ }^{55}$ y de la creación de controladores del saber ${ }^{56}$ "que haga vivir, cultive [...] sepa hacerse residencia" 57 . Atentos, intuitivos, los viajes y relatos de seminario sintomatizan, hasta ahora, sintonía con el avance general del saber y con la "ciencia de la complejidad": manifiestan la urgencia de cultivar los medios, recursos, condiciones para recorrer los caminos, para realizar los viajes del mundo saber-hospitalidad de lo cotidiano. De los viajes -" pasos del Nor-Oeste" Serresianos- atentos a las manifestaciones del "rostro" Levinasiano de la realidad.

\section{Bibliografía}

ANDERSEN, K; SOLÍS P. Memorias de la Cotidianeidad. Las huellas de lo intangible en la estructura tangible del lugar. Seminario, 2004.

ANDLER, D. Problème. Une clé universelle? En: I. Stengers (Ed). D'une science à l'autre. Des concepts nomades. Paris: Seuil, 1987.

ARAVOT, I. Back to Phenomenological Placemaking. Journal of Urban Design, 2002. 7, 2

ARAUJO, S; M-L. DE CAMARGO, R. "Phenomenology as a method to investigate the experience lived: A perspective from Husserl and Merleau-Ponty's thought". Journal of Advanced Nursing, 37, 3, 2002.

BACKHAUS, G. "Georg Simmel as an Eidetic Social Scientist". Sociological Theory, 16, 3, 1998.

BARNETT, C. "Ways of relating: Hospitality and the acknowledge of otherness". Progress in Human Geography, 29, 1, 2005.

BARRICO, A. Océano Mar. Barcelona: La Otra Orilla, 1993-1998.

BERTRAND, M. Planeamiento espacial estratégico colaborador y desarrollo local preventivo: De la hospitalidad. 2005.

BERTRAND, M. Caminos del urbanismo: Entre obligación y hospitalidad recorridos y reconfiguracion desde E. Lévinas. 2005

BOSCH, F. Planear. Megaproyectos Immobilarios como Tableros de Juego. Seminario, 2003.

BOUMANS, M.; BEAULIEU, A. "Foreword to Objects of Objectivity". Social Epistemology, $18,2-3,2004$

BRONFEN, E. "There's no place like home. The Aporia of Homecoming. The Wizard of $\mathrm{Oz}$ (Victor Fleming)". Parallax, 6, 3, 2000.

CASORZO, M.J. De la composición arquitectónica de los ritmos compositivos. Seminario, 2003.

CERTEAU, M. L'écriture de l'histoire. Paris: Folio Gallimard, 1975.

CHRISTIN, A-M. L'Image Ecrite ou la déraison graphique. Paris: Flammarion, 1995-2001.

CLINGERMAYER, J.C. "Heresthetics and Happenstance: Intentional and Unintentional Exclusionary Impacts of the Zoning Decisionmaking Process". Urban Studies, 41, 2, 2004
COURTINE-DENAMY, S. "Le Visage en Question. De l'image à l'éthique". La Différence, 2004.

COUCLELIS, H. "The certainity of uncertainity: GIS and the limits of geographic knowledge". Transactions in GIS, 7, 2, 2003

DELEUZE, G.; LE PLI. Leibniz et le Baroque. Paris: Les Éditions de Minuit, 1988

DELEUZE, G.; GUATTARI F. Mille Plateaux. Paris: Les Editions de Minuit, 1980.

DE LEÓN, A.M. Hacia el proyectar(se) o de los emocionares en la transición íntimo/público. Seminario, 2003.

DERRIDA, J. De l'hospitalité. Paris: Calman-Lévy, 1997.

DERRIDA, J. Apories. Paris: Galilée, 1996.

DICKSON, D. "Chronotopic Innovation: The Dialogic Constitution of Experience in Bakthin and Ralph Waldo Emerson". Dialogism, 5, 6, 2001

DONOSO, D. Los vacíos de la ciudad. Casos del triángulo central de Santiago. Seminario, 2003

DOSSE, F. L'Empire du Sens. L'humanisation des Sciences Humaines. Paris: La Découverte, 1995 y 1997.

DREYFUS, H.L. Ser en el mundo. Santiago: Cuatro Vientos, 1991-1996.

ELGIN, C.Z. "Creation as reconfiguration: Art in the advancement of science". International studies in the Philosophy of Science, 16, 1, 2002

ENCINA, R. Experiencia sensible del espacio en la obra de María Luisa Bombal. Seminario. 2003.

FARINELLI, F. "Did Anaximander ever Say (or Write) any Words?" The nature of Cartographical Reason Ethics, Place and Environment, 1, 2, 1998.

FÉDIER, F. Regarder Voir. Les Belles Lettres. 1995.

FEYERABEND, P. Contre la Méthode. Esquisse d'une théorie anarchiste de la connaissance. Paris: Eds. du Seuil, 1975-1979.

GARCíA, I. Espacios Públicos abiertos: Kilómetro cero-en el recorrido lento hacia la construcción de los escenarios de la cotidianidad. Seminario, 2002.

GASQUET, J. Cézanne. Paris: Editions BernheimJeune, 1925-2002.

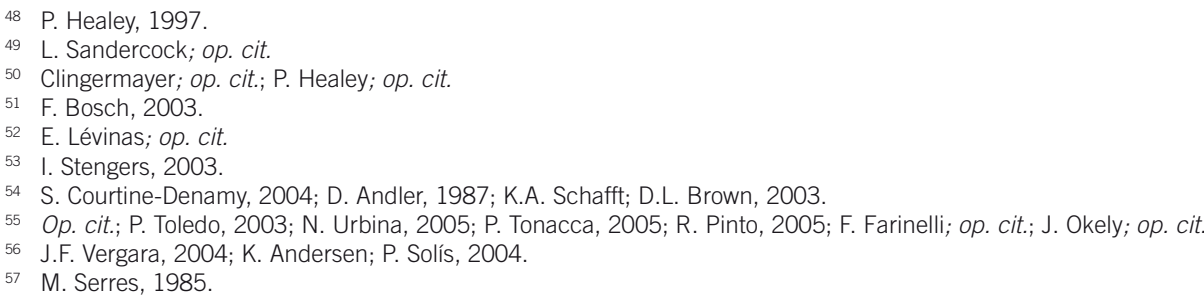




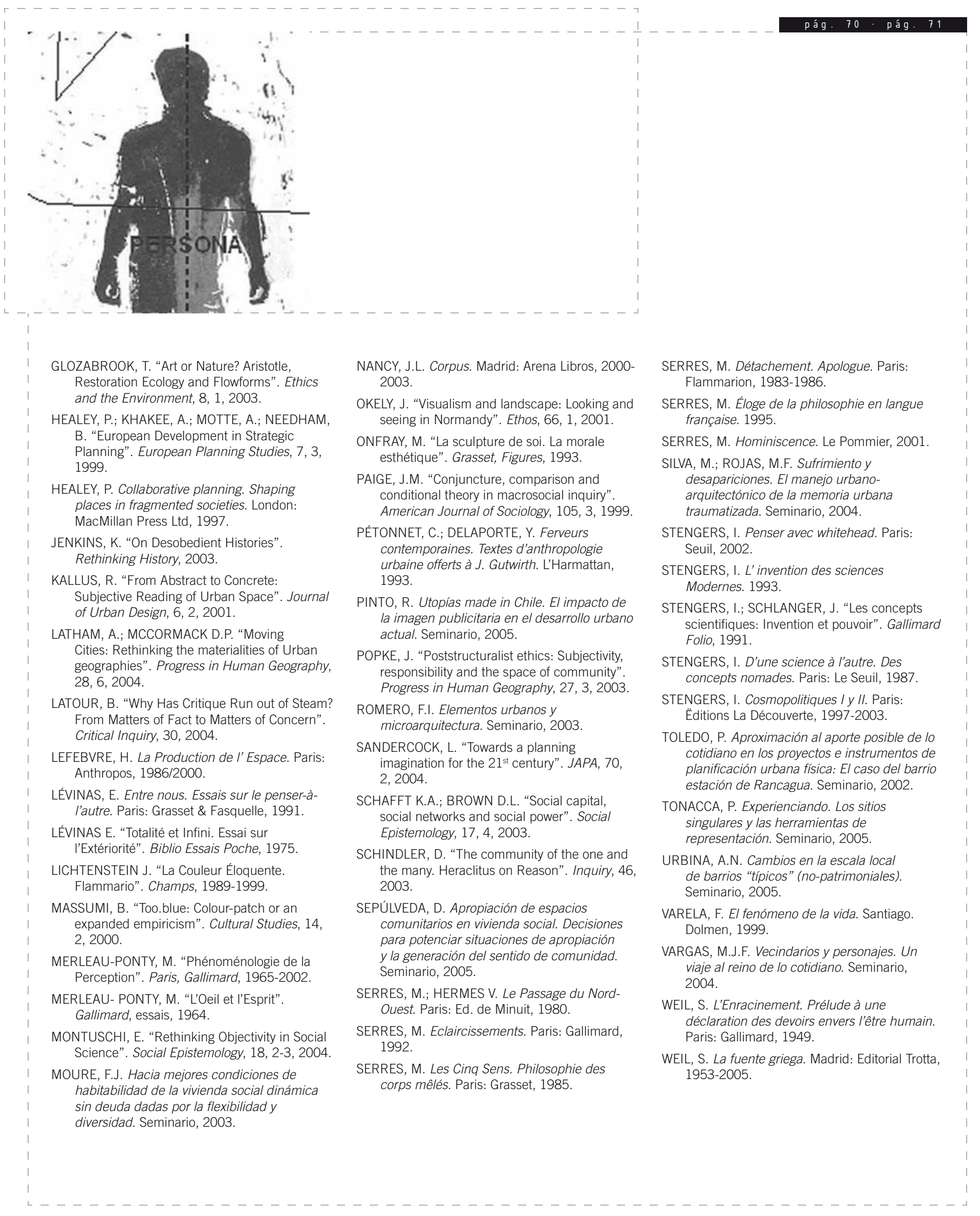

